\title{
The dosimetric impact of vaginal balloon-packing on intracavitary high-dose-rate brachytherapy for gynecological cancer
}

\author{
William M. Rockey, MD, PhD', Sudershan K. Bhatia, MD, PhD, MPH', Geraldine M. Jacobson, MD, MPH, MBA², \\ Yusung Kim, PhD' \\ IRadiation Oncology Department, The University of lowa, 2Radiation Oncology Department, West Virginia University, USA
}

\begin{abstract}
Purpose: We perform a clinical retrospective study to determine whether a vaginal balloon-packing system provides a dosimetric reduction to organs at risk (OARs) versus traditional gauze packing for gynecological high-dose-rate brachytherapy (HDR-BT). We also test various balloon filling materials for optimizing imaging quality.

Material and methods: Filling materials for balloon-packing were evaluated based on imaging quality with $X$-ray, computerized tomography, and magnetic resonance imaging modalities. We then retrospectively reviewed 45 HDR-BT plans of 18 patients performed with gauze packing and 39 plans of 16 patients performed with balloon-packing. Twelve patients received both gauze and balloon-packing. HDR-BT was delivered with an iridium-192 afterloader and a Fletcher-Suit-Declos-style T\&O applicator. At each fraction, 3D imaging was obtained. The $\mathrm{D} 2_{\mathrm{cc}}$ values of OARs were calculated, as well as ICRU-defined point doses.

Results: In the 84 HDR fractions reviewed, vaginal balloon-packing provides statistically equivalent doses to rectum, bladder, and sigmoid compared to gauze packing. On average balloon-packing produced average reductions of $3.3 \%$ and $6.9 \%$ in the rectal and sigmoid $\mathrm{D} 2_{\mathrm{cc}}$ doses and an increase of $3.2 \%$ to the bladder $\mathrm{D} 2_{\mathrm{cc}}$ dose (normalized to prescription dose), although none of these values were statistically significant for the twelve patients who received both gauze and balloon-packing (32 and 40 total fractions, respectively).

Conclusions: In the $84 \mathrm{HDR}$ fractions analyzed, vaginal balloon-packing is as effective as gauze packing for dose sparing to the rectum, bladder, and sigmoid. A $1: 1$ solution of saline and contrast for filling material enables easy contouring for image-guided HDR with minimal artefacts.
\end{abstract}

Key words: brachytherapy, gynecological cancer, high-dose-rate brachytherapy, vaginal balloon packing.

\section{Purpose}

Brachytherapy (BT) plays an essential role in the treatment of both endometrial cancer and carcinoma of the cervix [1,2]. The use of high-dose-rate (HDR) BT, typically with an iridium-192 remote afterloader, has become increasingly common for reasons of patient convenience, comfort, and expense $[3,4]$. The most commonly used HDR intracavitary applicators are the tandem-and-ovoids applicator $(\mathrm{T} \& \mathrm{O})$, and the tandem-and-ring applicator. The prescription dose calculation and dose calculation to the organs at risk (OAR) rectum and bladder are historically performed using the point-based Manchester system on orthogonal radiographs $[3,5]$, in which the radiation dose is prescribed to point $\mathrm{A}$, located $2 \mathrm{~cm}$ superiorly along the tandem from the lateral vaginal fornix and $2 \mathrm{~cm}$ laterally to the tandem $[6,7]$.

Traditionally, vaginal gauze packing has been used after applicator placement. This packing serves two impor- tant functions: immobilizing the applicator and increasing the distance between the radioactive source and the OARs, which subsequently decreases the OAR dose. Toxicities from gynecological BT manifest with a spectrum of clinical sideeffects ranging from urinary urgency rectal pain and rectal bleeding to chronic ulceration and vaginal fistulas. As an alternative to gauze packing, a commercial vaginal balloon-packing system was introduced that consists of two balloons which fit anteriorly and posteriorly over the BT applicator. There are several potential benefits to this system over traditional gauze-packing, including increased reproducibility among fractions, increased patient comfort, and a decreased risk of vaginal laceration during packing.

Three-dimensional (3-D) image-guided HDR-BT uses computed tomography (CT) or magnetic resonance imaging (MRI) to allow clinicians to verify proper placement of an applicator, and to allow for customized treatment plans to deliver dose to the tumor and spare OARs. The use of MRI 
in HDR-BT is currently gaining momentum $[8,9]$ due to its high soft tissue contrast, which provides better definition of the macroscopic tumor. Commonly referenced volumetric dose parameters include the $\mathrm{D} 2_{\mathrm{cc}}$, which is the dose in the "hottest" $2_{\mathrm{cc}}$ of a structure, as opposed to the traditional rectal and bladder point doses defined on 2-D imaging by the International Commission on Radiation Units and Measurements (ICRU) report \#38 [5].

While a previously published study used Monte Carlo modelling to show a theoretical $6-15 \%$ dose reduction to OARs using vaginal balloon-packing [10] due to the attenuating effect of a high CT-number contrast material used as filling, to our knowledge, no clinical studies concerning the dosimetry of vaginal balloon-packing have been reported. In this study, we investigate whether balloon-packing provides superior dose reduction to OARs compared with traditional gauze packing. We hypothesize that the increased reproducibility afforded by the balloon-packing system and improved treatment geometry would on average lead to decreased OAR doses compared to gauze packing, due to the inherent variability in quality of gauze packing. Toward this end, we describe a retrospective volume-based dosimetric analysis of OAR doses from HDR-BT plans performed with either gauze or balloon-packing. Additionally, dosimetric comparisons are made among fractions for patients who received both gauze and balloon-packing during the course of their treatment.

\section{Material and methods}

\section{Patients and treatment: EBRT and HDR}

Twenty-two patients with biopsy-confirmed cervical cancer who received HDR-BT were selected for this study. The use of patient data was approved by our Institutional Review Board. The patients' International Federation of Gynecology and Obstetrics (FIGO) stages ranged from IB2 to IIIB. Institutional standard-of-care treatment consisted of external beam radiation therapy (EBRT) with weekly chemotherapy (typically platinum based) integrated with HDR-BT. EBRT was $45 \mathrm{~Gy}$ in 25 fractions to the whole pelvis. Patients with parametrial involvement received an EBRT boost of $5.4 \mathrm{~Gy}$ in 3 fractions to the parametrium. After 3-4 weeks of EBRT, HDR-BT using a VariSource i $X^{\circledR}$ remote afterloading unit (Varian Medical Systems Inc., Palo Alto, California, USA) was started with a nominal 7 day interval between fractions. A titanium Fletcher-Suit-Delclos style $\mathrm{T} \& \mathrm{O}^{\circledR}$ (Varian Medical Systems Inc.) was used for all cases. The T\&O was inserted under general anesthesia after a clinical examination. Ultrasound guidance was performed for each $\mathrm{T} \& \mathrm{O}$ insertion.

After the applicator was inserted, a high-resolution (3.0 Tesla) MRI scan was acquired with a MAGNETOM Trio 3T MR ${ }^{\circledR}$ scanner (Siemens Medical Solutions Inc., Erlangen, Germany) with the use of both spine and body array coils. Detailed 3.0 Tesla MRI scan parameters can be found in a previously published study [11]. Other studies have examined the issues surrounding the use of titanium applicators in a high-resolution (3.0 Tesla) MRI scanner including safety, artefacts, and distortions [11,12]. Three HDR fractions in this study ( 3 out of 84 ) were planned using CT due to the unavailability of the MR scanner on the treatment day. Orthogonal digital radiographs, using a C-arm fluoroscopy unit (ACARDIS Orbit ${ }^{\circledR}$, Siemens Medical Solutions Inc.) were acquired in the HDR treatment room before and after the 3-D imaging scan (CT or MRI) to confirm that the applicators had not moved during patient transfer.

Our institutional standard HDR prescription scheme is 5.5 Gy times 5 fractions or 7 Gy times 4 fractions with 7 day intervals, integrated with EBRT so that the radiation course is finished within 7 weeks [3]. HDR treatment plans were performed based upon the conventional Manchesterbased system $[3,13]$, in which radiation dose is prescribed to point A. The BrachyVision version $8.9^{\circledR}$ (Varian Medical Systems Inc.) treatment planning system was used for HDR planning and dose volume histogram analysis.

\section{Vaginal packing procedure}

After the T\&O applicator was positioned, either gauze or balloon-packing (the Alatus ${ }^{\circledR}$ Vaginal Balloon Packing System, Radiadyne LLC., Houston, Texas, USA) was used to immobilize the applicator. For gauze-packing, a roll of gauze was soaked in sterile normal saline. Beginning at the proximal vagina, approximately $5 \mathrm{~cm}$ segments of gauze were inserted with forceps, alternating anteriorly and posteriorly to the applicator. Gauze insertion continued until the vaginal introitus was reached and the applicator remained snugly in place.

The balloon-packing system consists of two balloons which are inserted anteriorly and posteriorly to the applicator. A 50 cc syringe was filled with a solution of one part normal saline and one part ISOVUE-250 ${ }^{\circledR}$ contrast (Bracco Diagnostics Inc., Princeton, New Jersey, USA). This syringe was used to alternately fill the anterior and posterior balloons in $10 \mathrm{cc}$ increments. Each balloon was typically filled with $30 \mathrm{cc}$ to $50 \mathrm{cc}$ of solution depending on the vaginal size.

\section{Commissioning vaginal balloon-packing}

Various balloon filling materials were examined, including air, water, $100 \%$ contrast, $100 \%$ normal saline, and a $1: 1$ solution of normal saline and contrast (Fig. 1). The optimal filling material was determined using two metrics: favourable imaging qualities on orthogonal radiograph, CT, and MRI modalities (most importantly lack of artefact), and also radiation attenuation capacity. A quality assurance (QA) phantom, previously developed to perform QA for CT or MRI-guided HDR [11], was also used in this study. The Hounsfield Unit (HU) values on CT were quantified to estimate the radiation attenuation potential for the various filling materials. The vaginal balloon-packing material which best balanced imaging characteristics with the potential for dose attenuation was clinically implemented.

\section{Dosimetric evaluation}

To perform in vivo dosimetric evaluations, we retrospectively reviewed $45 \mathrm{HDR}$ plans of 18 patients that were performed with gauze packing and 39 plans of 16 patients performed with balloon-packing. Twelve patients received both gauze and balloon-packing. The rectum, bladder, and sigmoid were contoured on high-resolution (3.0 Tesla) MRI 

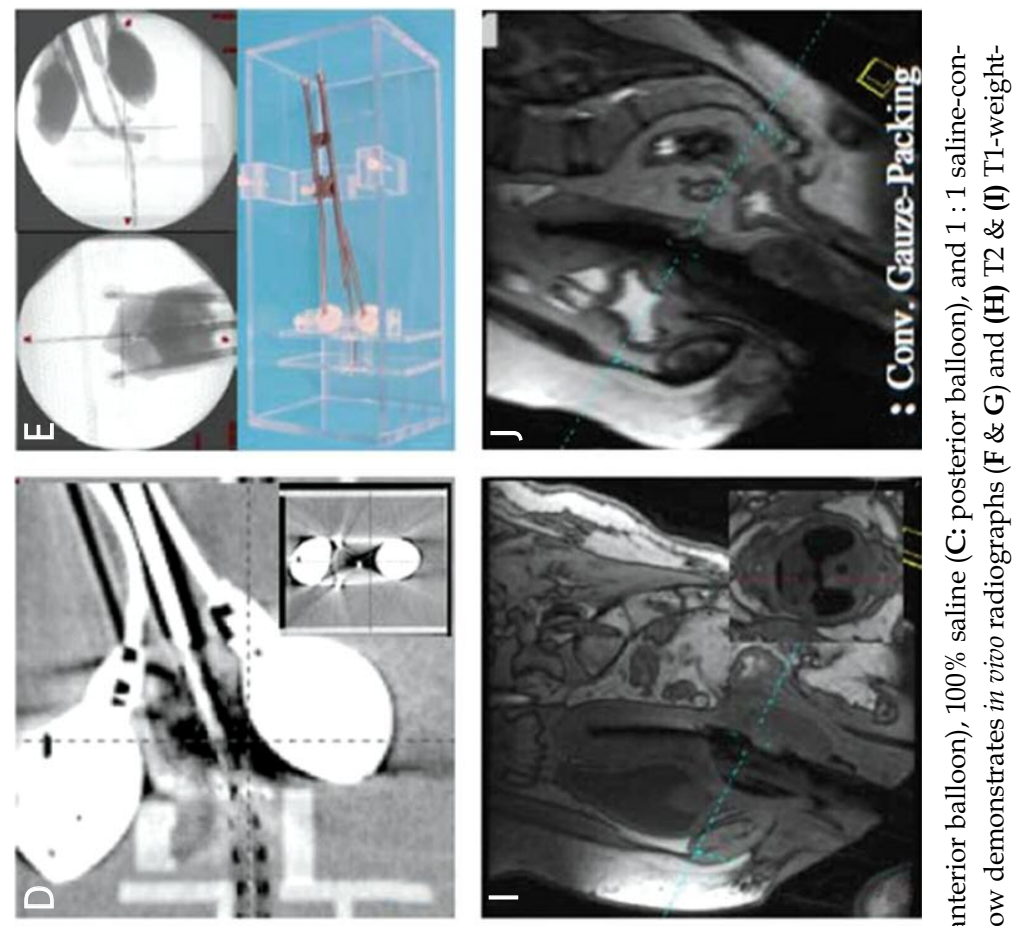

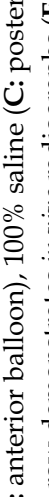
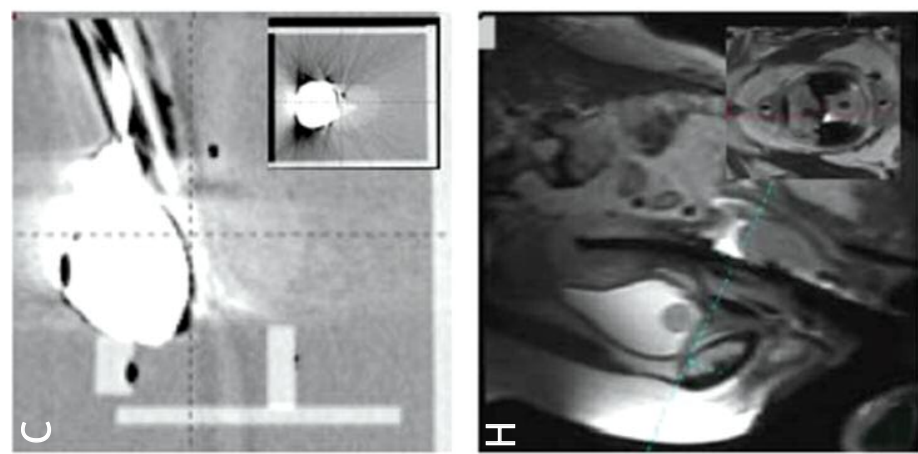

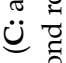

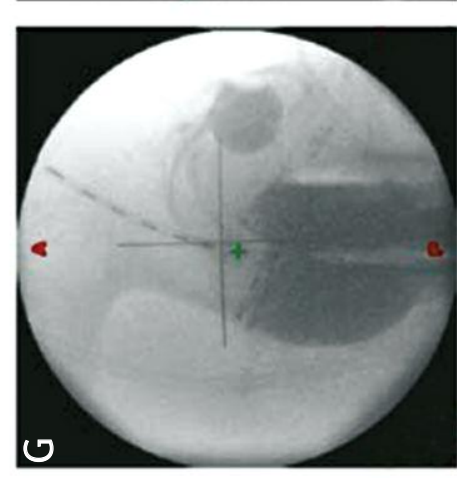

菏

茎艺

ஓ̊ ॄें

它

กิ

売岕

3

运

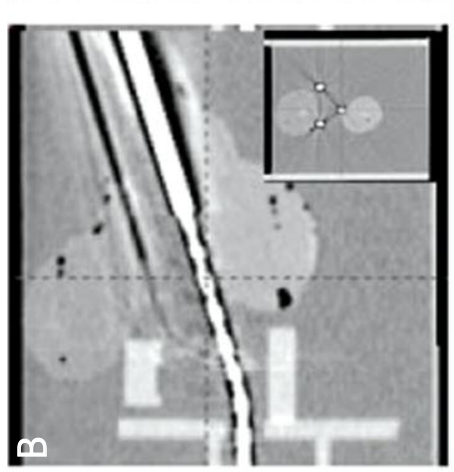

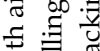

赍灵
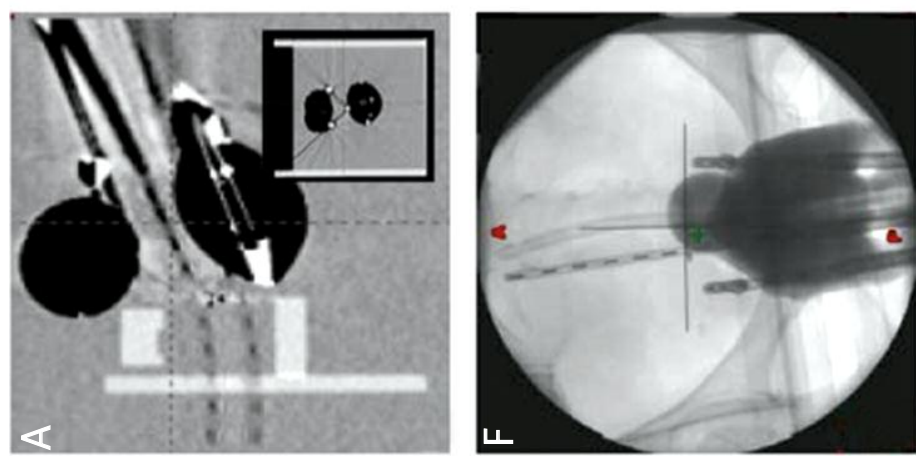

bo

政 3

苋 종

:

స్ 준

ฮี

漹

음

$30 \sum^{\circ}$

胥

छै की

已

का

造造

उह चै

을

क

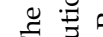

굴요

ab 
or CT scans, according to the GEC-ESTRO-EMBRACE guidelines [8]. Values for $\mathrm{D} 2_{\mathrm{CC}}$ of the OARs were used as metrics to compare the dosimetric impact of vaginal packing, along with conventional ICRU report \#38-defined rectal and bladder point doses. Both the ICRU point doses and the $\mathrm{D} 2_{\mathrm{cc}}$ values were converted to a percentageof-prescription dose for that fraction by dividing by the prescription dose and multiplying by 100 (referred to as $\%$ ICRU or $\% \mathrm{D} 2_{\mathrm{cc}}$. This was done to normalize to the prescription dose (5.5 Gy or $7 \mathrm{~Gy}$ ). The average ICRU, \% ICRU, $\mathrm{D} 2_{\mathrm{cc}}$ and $\% \mathrm{D} 2_{\mathrm{cc}}$ values for the OARs were calculated for gauze and balloon-packing, as well as standard deviations. The $p$-values were calculated using Microsoft Excel using a two-tailed $t$-test assuming homoscedasticity.

An "intra-patient" comparison of balloon and gauze packing was conducted for twelve patients who received both gauze and balloon-packing at different fractions during the course of their treatments. These patients each received 1-3 fractions packed with gauze and 1-4 fractions packed with balloons. The $\% \mathrm{ICRU}$ and $\% \mathrm{D} 2_{\mathrm{cc}}$ doses were calculated as described above for the ICRU point doses and the image-based $\mathrm{D} 2_{\mathrm{cc}}$ values, respectively. For those patients who received more than one fraction with a particular packing type, values were averaged and the standard deviation was calculated.

\section{Results}

\section{Balloon filling material}

We found that air filling was suboptimal for balloonpacking due to the loss of attenuation potential. Saline provided both a degree of attenuation $(62 \pm 32 \mathrm{HU})$ and satisfactory image quality on radiographs and CT, showing the vaginal wall clearly with no artefacts, and on T2-weighted MRI with high MR signal. Contrast used as filling produced a high HU (2790 \pm 585$)$, which was potentially promising for further attenuation to the OARs, but imaging artefacts were unacceptable (Fig. 1). A $1: 1$ mixture of saline and contrast produced acceptable imaging on orthogonal radiographs, CT, and MRI with a HU value of $1882 \pm 449$, also promising for potential attenuation. The $1: 1$ saline and contrast filling was selected for our institutional protocol for MRI-guided BT. Both radiographs and MRI (T1 \& T2 weighted) with balloon-packing showed the vaginal wall clearly, aiding with contouring during treatment planning.

\section{Dosimetric analysis}

A summary of the dosimetric comparison between balloon-packing and gauze packing is shown in Table 1. Balloon-packing provided a non-statistically significant reduction in the $\mathrm{D} 22_{\mathrm{CC}}$ rectal dose expressed as a percentage of the prescription (53.1\% balloon vs. $60.7 \%$ gauze, $p=0.07$ ). Likewise, the bladder and sigmoid D2 ${ }_{\mathrm{cc}}$ volumetric doses and the ICRU rectal and bladder point doses were statistically equivalent between balloon-packing and gauze packing. The bladder $\mathrm{D} 2_{\mathrm{cc}}$ dose was higher for balloon packing $(98.1 \%$ balloon vs. $87.7 \%$ gauze, $p=0.16$ ), but again, this was not statistically significant.

In order to account for anatomic differences, a comparison of OAR doses was made among fractions within the same patients, i.e., 12 patients who received both gauze and balloon-packing alternatively at different fractions during the courses of their treatments (Table 2). Each patient received 1-4 fractions of both packing types. For these twelve patients as a group, balloon-packing produced average reductions of $-3.3 \%$, and $-6.9 \%$ in the rectal and sigmoid D2 $2_{\mathrm{cc}}$ doses, and an increase of $+3.2 \%$ in the bladder $\mathrm{D} 2_{\mathrm{cC}}$ dose (expressed as a percentage of the prescription dose). There were $-3.5 \%$ and $-4.8 \%$ reductions in the rectal and bladder ICRU point doses as a percentage of the prescription doses. None of these values were statistically significant.

\section{Discussion}

Gauze has been utilized as vaginal packing for gynecological BT for decades with traditional 2-D orthogonal imaging. The quality of gauze packing is highly dependent on the experience and skill of the oncologist performing the procedure. Potential pitfalls include not packing tightly enough or an uneven distribution of gauze between the anterior and posterior parts of the applicator. At our institution, the applicator is placed while the patient is under general anaesthesia, but aggressive packing can be very uncomfortable for sedated patients. The vaginal wall is also susceptible to laceration during the packing process, causing further potential morbidity to the patient and increasing the total procedure time if sutures are required.

Balloon-packing addresses many of the problems with gauze packing. We hypothesized that balloon-packing would decrease dose to the OARs by increasing distance from sources and a more favourable geometry. Saini et al. [10] reported contrast-filled balloons showed $7.8 \%$ and $19.2 \%$ dose reductions through diode measurement when compared to saline- and air-filled balloons, respectively. Monte Carlo modelling also predicted $10.5 \%$ and $21.9 \%$ dose reductions in contrast-filled balloons, compared to saline- and air-filled balloons, respectively. In this study, ISOVUE-250 contrast was used in which iodine is the effective element and has $33 \mathrm{keV}$ K-shell electron binding energy. Thus, iodine has a high attenuation coefficient due to the photoelectric effect for low energy X-rays or gamma-rays. Enhanced photon absorption in the solution is, therefore, expected to be due to the large number of the photoelectric events, because of the presence of iodine. No study has been reported, validating dose reduction on OAR due to the use of contrast-filled balloon packing using clinical datasets. This is an area for future research.

In our analysis of $84 \mathrm{HDR}$ fractions, we did not find that balloon packing made a statistically significant difference in the bladder, rectal, or sigmoid OAR doses using either ICRU point criteria or a volumetric $\mathrm{D} 2_{\mathrm{CC}}$ analysis. Anatomical variation in these OARs may explain why there was not a significant reduction in doses to these structures with balloon-packing compared to gauze packing. This variation is seen among patients and also among fractions for the same patient. In an attempt to account for the former, we compared both packing types in twelve patients who received gauze and balloon-packing during the courses of their treatments. In this analysis, balloon-packing did produce a slight average reduction in dose for the rectum and sigmoid (3.3\% 
Table 1. Retrospective dosimetric analysis of 45 HDR brachytherapy plans performed with gauze packing and 39 plans performed with vaginal balloon packing. Mean values and standard deviations are shown below, as well as $p$-values from a two-tailed $t$-test. The average dose per fraction was similar between the gauze packing and balloon packing groups (6.1 Gy and 6.2 Gy). ICRU rectal and bladder point doses and the D2 ${ }_{\mathrm{cC}}$ values for the rectum, bladder, and sigmoid were calculated. \%ICRU and \%D2 $c$ refer to the ICRU and D2 $2_{C C}$ values as a percentage of the prescription doses

\begin{tabular}{|c|c|c|c|c|c|c|c|c|c|c|c|c|}
\hline \multirow{3}{*}{$\begin{array}{l}\text { Pre- } \\
\text { scription }\end{array}$} & \multicolumn{4}{|c|}{ Rectum } & \multicolumn{4}{|c|}{ Bladder } & \multicolumn{4}{|c|}{ Sigmoid } \\
\hline & Gauze & Balloon & $\Delta$ & $p$ & Gauze & Balloon & $\Delta$ & $p$ & Gauze & Balloon & $\Delta$ & $p$ \\
\hline & $6.1 \pm 0.7 \mathrm{~Gy}$ & $6.2 \pm 0.7 \mathrm{~Gy}$ & & 0.52 & $6.1 \pm 0.7 \mathrm{~Gy}$ & $6.2 \pm 0.7 \mathrm{~Gy}$ & & 0.52 & $6.1 \pm 0.7 \mathrm{~Gy}$ & $6.2 \pm 0.7 \mathrm{~Gy}$ & & 0.52 \\
\hline ICRU & $3.7 \pm 1.3 \mathrm{~Gy}$ & $3.3 \pm 1.2 \mathrm{~Gy}$ & -0.4 Gy & 0.14 & $4.4 \pm 1.9$ Gy & $4.3 \pm 2.2 \mathrm{~Gy}$ & $-0.1 \mathrm{~Gy}$ & 0.78 & & & & \\
\hline$\%$ ICRU & $60.7 \pm 19 \%$ & $53.1 \pm 18.4 \%$ & $-7.6 \%$ & 0.07 & $71.5 \pm 26.1 \%$ & $69.5 \pm 37.4 \%$ & $-2.0 \%$ & 0.78 & & & & \\
\hline $\mathrm{D} 2_{\mathrm{cc}}$ & $3.8 \pm 1.1 \mathrm{~Gy}$ & $3.5 \pm 1.2 \mathrm{~Gy}$ & $-0.3 \mathrm{~Gy}$ & 0.21 & $5.4 \pm 1.6 \mathrm{~Gy}$ & $6.1 \pm 2.6 \mathrm{~Gy}$ & +0.7 Gy & 0.14 & $3.7 \pm 1 \mathrm{~Gy}$ & $3.5 \pm 1.4 \mathrm{~Gy}$ & $-0.2 \mathrm{~Gy}$ & 0.31 \\
\hline$\% \mathrm{D} 2_{c c} 6$ & $62.4 \pm 14.2 \%$ & $56.5 \pm 17 \%$ & $-5.9 \%$ & 0.09 & $87.7 \pm 21.1 \%$ & $98.1 \pm 43.8 \%$ & $+10.4 \%$ & 0.16 & $61.6 \pm 14.6 \%$ & $55.9 \pm 20.4 \%$ & $6-5.7 \%$ & 0.15 \\
\hline
\end{tabular}

Prescription - prescriptions for all 45 HDR plans, Gauze - gauze packing data, Balloon - vagina balloon packing data, $\Delta$ - the mean difference of balloon-gauze (neg ative represents dose reduction at balloon), $p-p$ values, ICRU - ICRU report \#38 defined rectal and bladder point doses, \%ICRU and \%D2 $c C-I C R U$ and D2 $c C$ values as a percentage of the prescription doses

Table 2. The dosimetric differences of 12 intra-patients who received both gauze and balloon packing. Negative represents dose reduction in balloon packing

\begin{tabular}{|c|c|c|c|c|c|c|c|c|c|c|}
\hline & \multicolumn{4}{|c|}{ Difference in rectum } & \multicolumn{4}{|c|}{ Difference in bladder } & \multicolumn{2}{|c|}{ Difference in sigmoid } \\
\hline & $\begin{array}{l}\text { ICRU } \\
\text { [Gy] }\end{array}$ & $\begin{array}{c}\text { \%ICRU } \\
\text { [\%] }\end{array}$ & $\begin{array}{l}\mathrm{D} 2_{\mathrm{cc}} \\
{[\mathrm{Gy}]}\end{array}$ & $\begin{array}{c}\% \mathrm{D} 2_{\mathrm{cc}} \\
{[\%]}\end{array}$ & $\begin{array}{l}\text { ICRU } \\
\text { [Gy] }\end{array}$ & $\begin{array}{c}\text { \%ICRU } \\
\text { [\%] }\end{array}$ & $\begin{array}{l}\mathrm{D} 2_{\mathrm{cc}} \\
\text { [Gy] }\end{array}$ & $\begin{array}{c}\% \mathrm{D} 2_{\mathrm{cc}} \\
{[\%]}\end{array}$ & $\begin{array}{l}\mathrm{D} 2_{\mathrm{cc}} \\
\text { [Gy] }\end{array}$ & $\begin{array}{c}\% \mathrm{OD} 2_{c c} \\
{[\%]}\end{array}$ \\
\hline Patient 1 & -1.1 & -15.2 & -3.0 & -43.1 & -1.6 & -22.7 & -4.9 & -69.4 & -0.9 & -12.9 \\
\hline Patient 2 & +0.7 & +12.7 & +0.3 & +4.9 & -1.4 & -24.9 & +0.9 & +16.8 & -0.2 & -3.5 \\
\hline Patient 3 & -0.2 & -3.1 & +1.0 & +14.9 & -0.4 & -5.4 & +3.1 & +44.0 & +0.7 & +9.6 \\
\hline Patient 4 & -3.1 & -43.8 & -2.2 & -30.9 & -0.9 & -12.8 & -0.7 & -10.1 & -1.5 & -20.7 \\
\hline Patient 5 & -1.3 & -23.5 & -0.7 & -12.7 & -0.8 & -13.8 & -1.0 & -17.8 & -0.5 & -8.3 \\
\hline Patient 6 & +0.2 & +6.6 & -0.9 & -9.4 & +2.8 & +48.0 & +1.7 & +31.1 & -1.2 & -14.4 \\
\hline Patient 7 & +1.5 & +20.7 & +2.0 & +28.1 & -2.8 & -40.1 & -2.6 & -37.5 & +0.2 & +2.6 \\
\hline Patient 8 & +0.5 & +7.1 & +0.7 & +10.4 & +0.8 & +11.3 & +1.5 & +21.7 & +0.6 & +7.8 \\
\hline Patient 9 & -0.1 & -1.0 & -0.2 & -3.1 & -0.3 & -4.7 & +0.2 & +2.5 & -0.6 & -7.9 \\
\hline Patient 10 & +0.3 & +4.0 & 0.0 & +0.6 & +2.4 & +34.3 & +2.1 & +30.1 & -1.5 & -20.6 \\
\hline Patient 11 & -0.9 & -16.0 & +0.3 & +6.0 & -0.3 & -5.9 & +0.9 & +16.4 & +0.6 & +10.1 \\
\hline Patient 12 & +0.5 & +9.4 & -0.3 & -5.1 & -1.2 & -21.2 & +0.6 & +10.9 & -1.4 & -24.8 \\
\hline Average & -0.2 & -3.5 & -0.2 & -3.3 & -0.3 & -4.8 & +0.1 & +3.2 & -0.5 & -6.9 \\
\hline Std dev & 1.2 & 19.1 & 1.5 & 21.2 & 1.8 & 27.2 & 2.4 & 35.3 & 0.8 & 10.9 \\
\hline$P$ value & 0.52 & 0.48 & 0.58 & 0.55 & 0.75 & 0.70 & 0.85 & 0.74 & 0.26 & 0.31 \\
\hline
\end{tabular}

Difference - the difference of balloon packing data - gauze packing data, ICRU - ICRU report \#38 defined rectal and bladder point doses, \%ICRU and \%D2 $C C-I C R U$ and $D 2_{c c}$ values as a percentage of the prescription doses

and $6.9 \%)$, but an equally slight increase in dose to the bladder $(3.2 \%)$. These values were not statistically significant.

Among these twelve patients, many patients often had suboptimal high bladder doses for at least one fraction, regardless of the packing type (Table 2). Notably, the ICRU bladder point dose was often deceptively within normal limits ( $<80 \%$ of the prescribed dose), but the image-based $\mathrm{D} 2_{\mathrm{cc}}$ was over $100 \%$ of the prescribed dose. The disconnection between ICRU point doses and image-based dosimetry has been previously noted [14], and image-based dosimetry has been shown to be more clinically relevant in terms of side effects [15]. Our current study further underscores the im- portance of volume-based dosimetry in brachytherapy planning.

In a patient with a very anteverted uterus, portions of the bladder may be unavoidably near the tandem and therefore in the high-dose radiation isodose levels regardless of the type of packing used. Similarly, the anatomic location of the sigmoid colon varies among patients and among fractions, and its location with respect to the radiation isodose distribution is often unaffected by the type or degree of packing, since it is physically distant from the vagina.

When we started using balloon-packing in our practice, we filled both the anterior and posterior balloons to the no- 
minal $40 \mathrm{cc}$ as recommended by the vendor. Most patients in this study were treated according to these instructions. In contrast, when using gauze packing, the volume of packing is not set for each fraction, and the amount of packing a patient receives may vary from fraction to fraction based on the physician's skill and judgment. Nevertheless, we demonstrate that even with vendor recommended filling, we achieve, on average, comparable, if not better, dose sparing to OARs with balloon-packing. We believe that this demonstrates the major benefit of balloon-packing, which is a consistent quality of packing from fraction to fraction that minimizes the reliance on technique. We have recently begun to increase the filling volume of each balloon to $50 \mathrm{cc}$ if allowed by the patient's anatomy, which could conceivably provide some additional degree of dosimetric sparing to the OARs.

We show in this study that while there is not a dosimetric advantage to using balloon packing, an extensive analysis of 84 HDR fractions shows balloon packing provides statistically equivalent dosimetric protection to the OARs. This should provide reassurance to clinicians using balloon packing for reasons of patient comfort and convenience and may also suggest balloon packing as an appropriate selection for low-volume HDR centers with less experience with gauze packing. There are not enough patients and there has not been sufficient follow-up to know whether there are any differences in clinical outcomes between the two groups of patients. In the short term, we have not noted any significant differences in the acute side effect profiles.

\section{Conclusions}

To our knowledge, this is the first clinical analysis of the dosimetric performance of a commercially available vaginal balloon-packing system. We show that based on volumetric dosimetry, balloon-packing provides a statistically equivalent OAR protection in the 84 HDR fractions analyzed. Balloon-packing provides significant clinical benefits, including patient comfort and safety and time savings. Although we did not show that balloon packing is able to significantly lower OAR doses through geometry optimization, clinical demonstration of a possible dose reduction secondary to attenuation by a high-Z balloon filling material does remain an area for future research.

Despite the promise of using image-guided HDR to deliver more personalized, conformal radiation to patient's actual tumor volumes as opposed to the historical Manchester points, HDR delivery is ultimately constrained by the physical geometry of the implant, which in turn is dictated by the patient's anatomy and OAR constraints. New paradigms, including balloon-packing, offer additional tools for increasing the therapeutic ratio of brachytherapy, either by optimizing the geometry of the implant, or possibly through physical means such as introducing some degree of radiation attenuation in the balloon filling material.

\section{Acknowledgements}

This work was partially supported by research grants from Varian Medical Systems.

\section{References}

1. Hanks GE, Herring DF, Kramer S. Patterns of care outcome studies. Results of the national practice in cancer of the cervix. Cancer 1983; 51: 959-967.

2. Bialas B, Kellas-Sleczka S, Fijalkowski M et al. Tolerance and efficacy of preoperative intracavitary HDR brachytherapy in IB and IIA cervical cancer. J Contemp Brachytherapy 2009; 1: 38-44.

3. Nag S, Erickson B, Thomadsen B et al. The American Brachytherapy Society recommendations for high-dose-rate brachytherapy for carcinoma of the cervix. Int J Radiat Oncol Biol Phys 2000; 48: 201-211.

4. Palmer A, Hayman O, Muscat S. Treatment planning study of the 3D dosimetric differences between Co-60 and Ir-192 sources in high dose rate (HDR) brachytherapy for cervix cancer. J Contemp Brachytherapy 2012; 4: 52-59.

5. "ICRU-report-38". Dose and volume specification for reporting intracavitary therapy in gynecology. International Commission on Radiation Units and Measurements, Bethesda 1985.

6. Anderson J, Huang Y, Kim Y. Dosimetric impact of point A definition on high-dose-rate brachytherapy for cervical cancer: evaluations on conventional point A and MRI-guided, conformal plans. J Contemp Brachytherapy 2012; 4: 241-246.

7. Tod M, Meredith W. A dosage system for use in the treatment of cancer of the uterine cervix. Br J Radiol 1938; 11: 809-824.

8. Haie-Meder C, Pötter R, Van Limbergen E et al. Recommendations from Gynaecological (GYN) GEC-ESTRO Working Group (I): concepts and terms in 3D image based 3D treatment planning in cervix cancer brachytherapy with emphasis on MRI assessment of GTV and CTV. Radiother Oncol 2005; 74: 235-245.

9. Pötter R, Haie-Meder C, Van Limbergen E et al. Recommendations from gynaecological (GYN) GEC ESTRO working group (II): concepts and terms in 3D image-based treatment planning in cervix cancer brachytherapy-3D dose volume parameters and aspects of 3D image-based anatomy, radiation physics, radiobiology. Radiother Oncol 2006; 78: 67-77.

10. Saini AS, Zhang GG, Finkelstein SE et al. Dose reduction study in vaginal balloon packing filled with contrast for HDR brachytherapy treatment. Int J Radiat Oncol Biol Phys 2011; 80: 1263-1267.

11. Kim Y, Muruganandham M, Modrick JM et al. Evaluation of Artifacts and Distortions of Titanium Applicators on 3.0-Tesla MRI: Feasibility of Titanium Applicators in MRI-Guided Brachytherapy for Gynecological Cancer. Int J Radiat Oncol Biol Phys 2011; 80: 947-955.

12. Nixon E, Kim Y, Kearney WR et al. HDR Brachytherapy Tandem and Ovoid Titanium Applicator Safety Assessment in 3T MRI. Brachytherapy 2008; 7: s135 (abstract).

13. Measurements ICoRUa. ICRU report 38: Dose and volume specification for reporting intracavitary therapy in gynecology. International Commission on Radiation Units and Measurements, Bethesda 1985.

14. Patil VM, Patel FD, Chakraborty $S$ et al. Can point doses predict volumetric dose to rectum and bladder: a CT-based planning study in high dose rate intracavitary brachytherapy of cervical carcinoma? Br J Radiol 2011; 84: 441-448.

15. Georg P, Kirisits C, Goldner G et al. Correlation of dose-volume parameters, endoscopic and clinical rectal side effects in cervix cancer patients treated with definitive radiotherapy including MRI-based brachytherapy. Radiother Oncol 2009; 91: 173-180. 